\title{
Reliability and Energy Efficiency Enhancement in Wireless Body Area Networks for E-Health
}

\author{
Marwa Salayma, Ahmed Al-Dubai, Imed Romdhani \\ Edinburgh Napier University \\ Edinburgh, United Kingdom \\ m.salayma, a.al-dubai, i.romdhani@napier.ac.uk
}

\begin{abstract}
Wireless Body Area Network (WBAN) is a technology that revolutionises our understanding of the future digitised healthcare systems. WBAN has unique challenges and features compared to other wireless sensor networks. In addition to battery power consumption, the vulnerability and the unpredicted channel behaviour make channel access a serious problem to solve. Time Division Multiple Access (TDMA) Medium Access Control (MAC) protocols can help in achieving a reliable and energy efficient WBAN. IEEE 802.15.4 provides TDMA based mechanisms to save energy consumption. However, both contention-free and inactive periods are static and do not consider channel status or nodes reliability requirements. Hence, this paper presents two IEEE 802.15.4 TDMA based techniques to improve WBAN reliability and energy efficiency. The first technique allows nodes to avoid channel deep fade by distributing adaptively their sleep period during their active period according to their channel status. Thereafter, in the second technique, nodes are dynamically allocated time slots according to their requirements, which depend on their link's status. The proposed techniques are evaluated over various traffic rates and their performances are compared with the legacy IEEE 802.15.4 MAC. Results revealed that the proposed techniques have the potential in improving WBAN reliability while reducing energy consumption.
\end{abstract}

\section{INTRODUCTION}

During the $20^{\text {th }}$ century, a mighty shift towards replacing wired sensors with wireless ones forming a wireless network of sensors from which the Wireless Sensor Network (WSN) era had emerged. It's not so long when such a revolution has shifted its direction to a technology that suits human mobility by surrounding human body with wearable and implanted, low cost, energy constrained tiny and heterogeneous sensor nodes. These nodes form what we call nowadays Wireless Body Area Network (WBAN). WBAN comprises sensors that capture body physiological information and send it to a central base station through wireless communication. WBAN replaces complex and wired healthcare equipment to perform continuous monitoring of vital information without limiting an individual's movements [1]. Similar to other communication networks, WBAN is vulnerable due to its resource-constrained nodes and to the harsh environment that surrounds them [2]. However, unlike other communication networks, WBAN abnormality might lead to life-threatening situations. Thus, it is important to guarantee that a WBAN works properly despite abnormalities that might occur at runtime. WBAN sensing devices are battery powered and resource constrained devices, which makes energy depletion a serious problem. Additionally, the human body absorbs energy when it is exposed to Radio Frequency (RF) electromagnetic, which weaken the signal [3]. Transmission paths are also affected by the uncontrolled, dynamic nature of the human body movement [4][19]. Those reasons cause continuous attenuation in WBAN channels. Consequently, the signal falls below receiver sensitivity and causes deep fade phenomena. Channel deep fade adversely affects WBAN reliability because it might disconnect the sensor from the network up to several minutes, which increases data loss and end-to-end delay and could lead to deadly situations [16][17][18]. According to the empirical study presented in [16] which's results are based on a real WBAN worn by a person doing different daily-activities, it is revealed that when a deep fade occurs in the WBAN channel, it lasts for at least $10 \mathrm{~ms}$. This slow changing channel characteristic can be exploited to predict the channel performance in upcoming time-periods. For instance, if by some way a node knows that the link between himself and the controller is vulnerable due to deep fade at a given time, it can avoid direct re-transmission. This is because the deep fade in the link is most likely to persist for long time, and performing re-transmission is therefore useless. If nodes are allowed to operate in a timely, scheduled manner, following contention free channel access, such as a TDMA approach, then fading becomes the primary source of unreliable WBAN. This will not only help in channel status prediction, but will also save energy because contention free techniques avoid idle listening and overhearing.

TDMA techniques provide static slot allocation, which means that a node will stay awake and will keep on transmitting in its time slot regardless of the occurrences of the deep fade in the links between the controller and the nodes. If a node's link is in a bad state during its time slot due to deep fade, node packets loss will continue, which will not only decrease packets reception rate and decreases reliability but also will dissipate energy. How to cope with this problem is the aim of our proposed protocol in this study. The proposed protocol is a TDMA based channel access mechanism, which harnesses the slow changing channel behavior in re-allocating channel slot adaptively while saving energy. 
IEEE 802.15.4 is one of the WBAN de-facto standards that provides the capability of TDMA channel access mechanisms through the Guaranteed Time Slots (GTS(S)) provided in the Contention Free Period (CFP) in the beacon-enabled mode. It also offers nodes the option to save extra energy through the inactive period [26]. However, if they are enabled, both the CFP and the inactive periods are static and do not consider anything related to the network, for example, the channel status. Although existing schemes provide some solutions to providing reliable WBANs [2][6][7][9-15], designing efficient generic WBAN protocols that are resilient to channel impairments is still a challenging issue. In addition, the majority of the proposed protocols require complex amendments to the MAC specification of the WBAN standards (i.e., IEEE 802.15.4 and IEEE 802.15.6). This motivates to tackle WBAN from the MAC layer angel and design a channel-access mechanism, which is based on an empirical channel-data that is able to improve WBAN reliability by taking into account the channel status. The proposed methods consider avoiding fading, energy efficiency and dynamic channel allocation. The proposed techniques were compared to the legacy beacon enabled IEEE802.15.4. The considered performance metrics are reliability, timeliness and energy efficiency. Results revealed that the proposed techniques achieve a more reliable and energy efficiency than the legacy IEEE802.15.4 MAC.

The rest of the paper is structured as follows. The second section explores the literature review and critically review the most relevant and up-to-date proposed techniques. The third section presents the motivation behind the proposed techniques. The fourth section presents the proposed techniques along with their performance evaluations. The last section concludes our contribution and discusses future directions.

\section{RELATED WORK}

There is a number of existing MAC schemes in the literature that address the reliability issue in WBANs. The study in [5] surveys multiple contention and contention-free based MAC protocols from energy efficiency and TDMA perspectives [20-25]. This section illustrates those solutions and focus in particular on those that are closely related to our proposed techniques. Tselishchev et al. [10] proposed a dynamic synchronisation TDMA based technique, called a flipping algorithm to improve the performance of IEEE 802.15.6 free access mechanism. The hub observes links performance on the previous superframe and schedules nodes on the upcoming superframe accordingly. Those nodes whose links are observed good in the previous supeframe are synchronised before the nodes of bad links. Yet, the order of nodes with good links in accessing the channel is flipped according to when their links status was observed. The advantage of flipping is to harness the good status of the link as fast as possible before it goes to a bad state. The flipping technique provides a slight improvement over the static scheduling algorithm as it reduces packet loss by up to 17 percent. However, when it decides that the link is poor, the hub assumes that the link is at the beginning of its deep fade. Moreover, links might be at the beginning or at the end of the deep fade, which means that the flipping technique might not contribute on any improvement, and it might make things rather worse. In active networks, packets will be stored in the buffer which will increase packets delay, and at the end will cause buffer over flaw. Besides, this technique is fully centralized and nodes know nothing about their links status. To improve the performance of their protocol, Tselishchev et al. proposed an extension to their flipping algorithm by developing an adaptive re-transmission policy for the lost packets in the previous superframes. In [11], Tselishchev et al. suggest that packet re-transmission should be delayed to the subsequent superfames because direct packet retransmission is useless and will be at the expense of energy when there is fading at the current superframe. Tselishchev et al. in [11] also suggest a larger number of allocation slots than the number of nodes in the network, and thus having extra slots dedicated for packets retransmission. Extra slots are adaptively allocated anywhere in the superframe according to energy budget of the nodes. 'However, due to the unknown exact time of the occurrences of the deep fade, re-transmitted packets will still suffer from the same deep fade problems as the original packets and therefore will not guarantee successful packets delivery, which will still be an issue at the expense of power consumption. Yan and Liu in [7] proposed a hybrid superframe based MAC protocol (CA-MAC) which comprises three parts: the beacon frame, the contention-based period that adopts the slotted CSMA/CA and which changes dynamically according to the level of fading in the channel, and the third part is the contention free period, which follows the TDMA mechanism. CA-MAC allocates transmission bandwidth, according to the traffic context and the channel status respectively.

To relax the effect of the channel deep fade, nodes are allowed to access the channel during the contention-based period by following the CSMA/CA. Although the proposed algorithm can tolerate the context of the network in terms of the channel and the traffic contexts, it still does not guarantee reliable data transmission in real time entirely due to the collision in the network in the contention-based period. Although the contention process can distribute channel access between nodes, it does not avoid or mitigate channel fading. Thus, packets still will be lost not due to one reason but, rather, due to two reasons especially at the current superframe which is also at the expense of power consumption. Moreover, the performance of the proposed protocol is applied on a network of just three nodes, which is not enough to prove the resilient of the proposed protocol. In addition, the proposed technique is fully centralised as the coordinator performs the entire traffic and channel awareness procedure; thus, it is prone to a single point of failure.

Rezvani and Ghorashi in [12] proposed a protocol that supports the heterogeneous characteristics of different applications by separating medical and non-medical applications and splitting that traffic into normal and emergency medical traffic. The separation between nonmedical, normal medical, and emergency medical traffic is adaptive, according to channel condition and the user's medical situation. Medical data follow TDMA synchronization, while non-medical data follow contention- 
based access mechanism. The interval between consecutive transmissions changes adaptively according to the condition of channel deep-fading. The proposed algorithm outperforms both IEEE 802.15.4 and IEEE 802.15.6 MAC in terms of reliability, and power consumption. However, it might take three to four superframes for the emergency data to be transmitted. Consequently, emergent data will not be remitted in real time and it might suffer from long delay. Besides, when the channel deep fade is detected at the current superframe, the location of the Normal TDMA (NTDMA) period is changed to the end of the next superframe. This does not guarantee avoidance or mitigation of fading effect, and when a node link faces fade during its time slot, it is supposed to continue retransmission, which will increase packet loss. Moreover, due to the availability of contention-based periods, the proposed technique is still not reliable because nodes might lose packets due to collision besides consuming more energy. In addition, context awareness computation is centralised and requires a complex and overall change in the structure of both the IEEE 802.15.4 and IEEE 802.15.6. Apparently, designing efficient generic WBAN MAC protocols that are resilient to channel impairments is still a challenging issue. Despite a number of works that have tackled channel fading in WBAN, their improvements are not significant and no work so far could overcome or mitigate effectively channel deep fade problem. Indeed, the majority of the proposed protocols do require amendments to the structure of the proposed WBAN standards (IEEE 802.15.4 and IEEE 802.15.6).

\section{MOTIVATION}

To save energy, IEEE 802.15.4 provides an optional inactive period at the end of the superframe structure (the beacon interval). If it is enabled, the inactive period is fixed and static during which all nodes, including the PAN Coordinator (PANc) sleep all at once at the same time portion. This helps in reducing energy consumption, and allows nodes to sleep blindly without considering anything related to the status of the network. However, instead of allowing nodes to sleep all at once at fixed time portion at the end of the superframe, nodes themselves can choose when to sleep whenever it is suitable or necessary. Besides saving energy, in WBAN, the necessity is to achieve the highest possible reliability. Reliability is extremely affected by channel vulnerability due to channel deep fade, which could last for at least $10 \mathrm{~ms}$ [16-18]. This calls for allowing nodes to choose when to sleep according to their link status with the PANc. The question is therefore: how the resource constraint nodes can be smart enough to detect their link status during packet transmissions? Indeed, the study in [5] reveals that the contention frees access based MAC protocols achieves a more reliable and energy efficient WBAN than the contention based protocols. Accordingly, if nodes are allowed to follow just a TDMA based channel access mechanism, then a node can realise that the primary reason behind not receiving acknowledgment frame from the PANc after its packets transmission. This reason is the deep fade in the link between it and its PANc. Other reasons, such as packets collisions, would be unlikely because the nodes follow contention free channel access mechanism. Once it detects the occurrences of a channel deep fade and due to the slow and stable channel status, a node realises that there would be no benefit in performing packets re-transmission, and staying awake in this case would be a waste of energy.

More importantly, a node is not able to estimate when its link status has deteriorated, i.e. whether the deep fade has just started, in the mid of its duration or it is about to end its poor phase. Therefore, it would be more reliable and energy efficient for the node to shut down its transceiver and sleep for the rest of its assigned GTS(s) in case it does not receive acknowledgment from the PANc. The node can wake up again to receive the subsequent beacon frame from the PANc as usual. Following this approach, nodes need no longer sleep blindly all at once at the end of the IEEE802.15.4. They would rather choose to sleep adaptively according to the status of the links between them and the PANc. Moreover, nodes would be capable of detecting the channel status without the need of the supervision of the PANc and consequently. In this case, the nodes would be able to take decisions without the need of extra control packets or computational complexities from the PANc, which is sufficient in a highly vulnerable WBAN channel. It is worth stating that allowing the nodes to sleep adaptively does not mean that they cannot harness the static inactive period offered at the end of the IEEE 802.15.4 superframe. Besides sleeping adaptively during their active period, nodes can still be parametrised to sleep statically during the inactive period for the aim of saving more energy. Adaptive sleep during the active period according to channel status, on one hand will improve WBAN reliability while on the other hand will improve energy efficiency. This motivated proposing the Adaptive Sleep IEEE 802.15.4 MAC technique which is explained in subsection IV-A.

\section{THE PROPOSED TECHNIQUES}

This section illustrates each one of the two generic TDMA based MAC techniques.

\section{A. Adaptive Sleep IEEE 802.15.4 MAC}

IEEE 802.15.4 provides a TDMA based free channel access mechanism through the GTS(s) offered in the Contention Free Period (CFP), which follows the CAP (Contention Access Period) in the active period. By following the legacy IEEE802.15.4, nodes are assigned GTS(s) statically when the network is established according to the network configurations. This works as follows: After successfully associated with the PANc, nodes send GTS request packets through which they ask the PANc to assign them a number of GTS(s).

When the PANc receives the request, it acknowledges the node and offers that node a number of GTS(s) equal to what is presented in the GTS request. Nodes are scheduled to access the channel when the PANc receives the GTS request. The node with a request that has been accepted first will use the channel before other nodes. According to the schedule, a node will wake up during its GTS(s) period and try to transmit its packets. If the node fails to receive an acknowledgment frame from the PANc it presumes that its link with the PANc suffers from a deep fade, and thus will not perform packet re- 
transmission, but will shut down its transceiver instead, if the inactive period is enabled. In addition, the node will continue sleeping until the current BI finishes. The node will wake up when the current BI (Beacon Interval) finishes in order to receive the next beacon frame as usual. Other nodes will use the channel according to the schedule. We call this simple strategy Adaptive Sleep IEEE 802.15.4 MAC and Algorithm 1 shows its major steps.

1) Simulation setup and performance evaluation: The feasibility of the Adaptive Sleep IEEE 802.15.4 in improving the reliability and energy efficiency of WBAN is assisted by evaluating its performance against the legacy IEEE 802.15.4 MAC. For this purpose, we used the last version of Castalia open source simulator that is Castalia-3.3 [27]. Table I shows the simulation parameters used throughout our simulations.

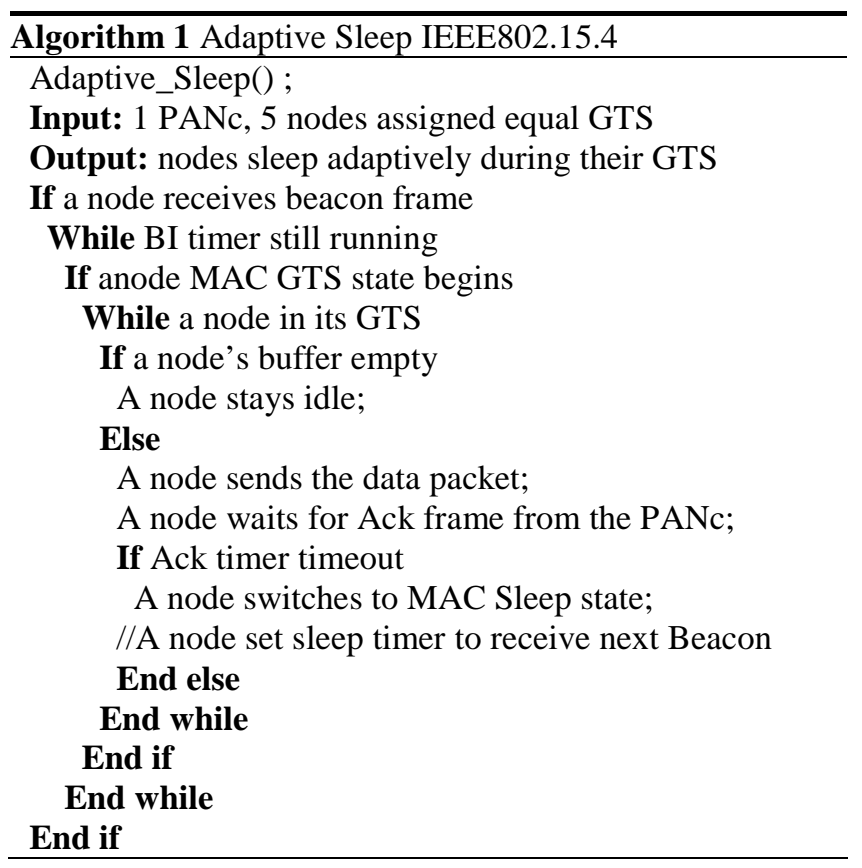

TABLE I. SIMULATION PARAMETERS

\begin{tabular}{cc}
\hline \hline Parameter & Value \\
\hline Simulator & Castalia 3.3 \\
Physical and MAC model & IEEE 802.15 .4 \\
Simulation time (s) & 500 \\
Number of nodes & 6 (1PANc and 5 nodes) \\
Tx power (dBm) & -15 \\
GTS(s) & 15 each node assigned 3 GTS \\
Buffer size (byte) & 32 \\
Traffic model & CBR (normal traffic) \\
Low & 1,5 \\
Moderate & 10,20 \\
High & 50 \\
Very high & 100 \\
Traffic rate (p/s) & 105 \\
Payload size (byte) & 6 \\
BO value & 4,5 \\
SO Values & \\
\end{tabular}

\section{Fig.1. Simulation topology}

Experiments run on a topology of 6 nodes, one PANc at the right hip, and five nodes (four at the four limbs and one in the middle of the chest) as depicted in Fig. 1. The study in [18] shows statistics about the duration of the deep fade for each node's link and how frequent does the signal falls below receiver sensitivity for that link. The 5 nodes send 105 bytes data packet (including overhead) to the PANc at a Constant Bit Rate (CBR) and 6 increasing traffic rates were tried. We evaluated the performance of our technique against the IEEE 802.15.4 beacon enabled MAC. There are 16 total superframe slots. In order to allow the nodes to follow only a TDMA based channel access, the 16 time slots are needed to be divided equally between the 5 nodes, and thus, each node will acquire 3 GTS. The remaining slot will be used by following a contention based channel access mechanism, i.e., the slotted CSMA/CA mechanism (according to [8][26], at least one slot should be given to contention based access). Each experiment was executed 5 times with different random seeds. Two scenarios were checked, which are presented in the following subsections.

2) Scenario 1: 25\% duty cycle Legacy IEEE 802.15.4 versus 50\% duty cycle Adaptive_Sleep IEEE 802.15.4 MAC: Our aim is to evaluate the gain of achieving a reliable WBAN by allowing nodes to sleep during their active period dynamically according to their links status, instead of sleeping statically and blindly at the end of the superframe. This means, we have to deactivate the inactive period by allowing nodes to operate within $100 \%$ (the value of Beacon Order (BO) and Superframe Order (SO) should be equal), and compare this configuration to a WBAN that follows the legacy IEEE 802.15.4 MAC but whish's inactive period is activated. To achieve this aim, for the legacy IEEE 802.15.4 MAC, we allowed the nodes to operate within $25 \%$ duty cycle and sleep for the rest of the $75 \%$ of the superframe structure $(\mathrm{BO}=6, \mathrm{SO}=4)$. However, for the Adaptive Sleep IEEE802.15.4, to save more energy, we thought to activate the inactive period for a while besides allowing nodes to sleep during their active period. Thus, we allowed nodes to operate within a $50 \%$ duty cycle and sleep for the rest of the $50 \%$ duty cycle of the superframe $(\mathrm{BO}=6$, $\mathrm{SO}=4$ ). This means that we presume that the rest of the $25 \%$ sleep period will be distributed during the nodes active period dynamically according to nodes links status with the PANc. The performances of both MAC algorithms are examined over various traffic rates, which range from low, moderate, high and very high traffic rates, as shown in Table I. 


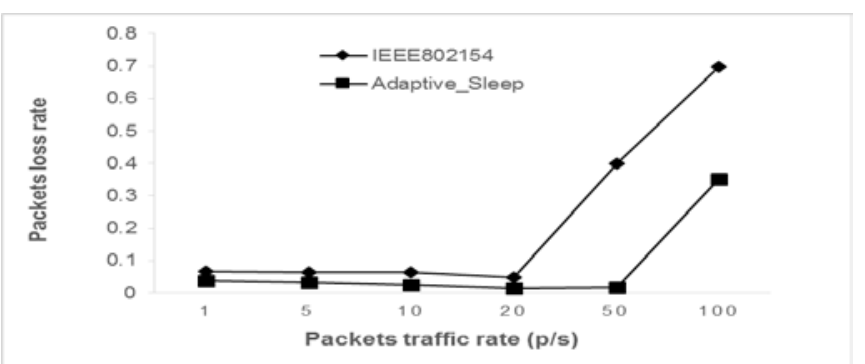

Fig. 2. Packets loss for an increasing packets rate

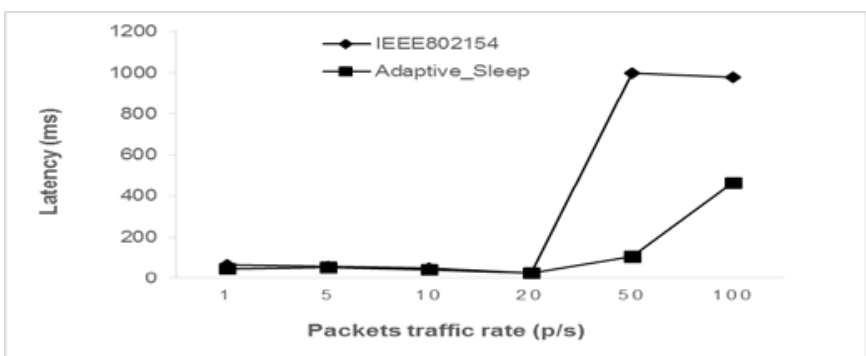

Fig. 3. End-to-end delay for an increasing packets rate

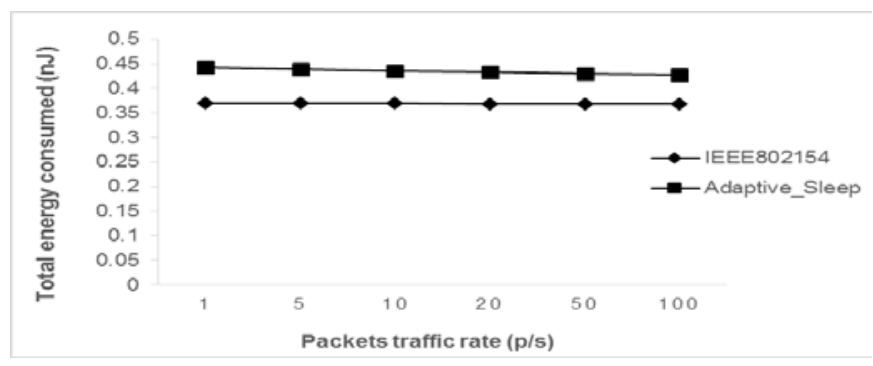

Fig. 4. Total consumed energy for an increasing packets rate

Both the legacy and the Adaptive Sleep IEEE802.15.4 MAC are compared against their reliability, which is measured in terms of the packets loss rate as illustrated by Fig. 2. It is obvious from Fig. 2 that Adaptive Sleep IEEE 802.15.4 MAC outperforms the legacy MAC at all the traffic rates. This is because the new technique avoids packets re-transmission when fading is detected. Consequently, it avoids the loss of more data packets. The improvement of the proposed algorithm is significant at high and very high traffic rates. This is because by following the legacy MAC, nodes sleep $75 \%$ of the superframe structure, whereas following the Adaptive_Sleep, nodes sleep for $50 \%$ during the inactive period and the possibilities for the deep fade is unlikely to occupy $25 \%$ of the active period of the superframe for each node. Thus, in general, the proposed algorithm offers nodes a shorter sleep period. Data packets that are received while the node is sleeping will be queued or saved in a temporary memory (buffer) until the node's next time schedule starts. However, due to the limited size of the buffer, packets will be dropped when the buffer become full. This is called buffer overflow. Long sleep periods cause buffer over flow at high (and very high) data rates, this explains the increase in packets loss at high traffic loads when nodes follow the legacy IEEE 802.15.4 MAC.

When it comes to timeliness, Fig. 3 reveals that Adaptive Sleep IEEE 802.15.4 MAC outperforms the IEEE 802.15.4 MAC at all traffic rates. This is due to the shorter sleep time portion offered by the Adaptive Sleep 802.15.4 MAC. Fig. 3 shows that Adaptive Sleep IEEE 802.15.4 increases latency at very high traffic rates, i.e. at $100 \mathrm{p} / \mathrm{s}$, this is because more packets will be saved in the node's buffer which will be prone to more delay. The legacy IEEE 802.15.4 on the other hand, causes an extreme buffer over flow at the very high traffic rates due to the longer sleep period it offers and the limited size of the buffer, therefore most of the packets will be lost leading to a latency performance close to that of high traffic rate, i.e. $50 \mathrm{p} / \mathrm{s}$.

However, as illustrated in Fig. 4, the new algorithm consumes more energy than the legacy one, this is rational as Adaptive Sleep technique allows nodes to sleep less time portion, and thus nodes will have more opportunities to use the channel which will consumes more energy.

3) Scenario 2: 50\% duty cycle Legacy IEEE 802.15.4 versus 50\% duty cycle Adaptive Sleep IEEE 802.15.4 MAC: Results achieved in subsection IV-A-2 call for conducting a fairer performance evaluation between the two MAC protocols. Both the legacy IEEE 802.15.4 and the Adaptive Sleep IEEE 802.15.4 MAC are required to operate within the same duration of the active period, i.e. both should acquire a $50 \%$ duty cycle and sleep 50\%. However, the nodes in the Adaptive Sleep MAC are allowed to sleep further during their active periods according to their channel status. Consequently, the proposed algorithm offers the nodes the opportunity to sleep longer than the legacy IEEE802.15.4. The two algorithms are simulated following the same parameters depicted in Table I and both MAC protocols are compared against the same performance metrics presented in subsection IV-A-2. Results are given in the Fig. 5-7.

Fig. 5 shows that Adaptive Sleep IEEE 802.15.4 MAC achieves less packets loss for all data packets rate apart from the very high data rates applications due to buffer overflow and not to channel vulnerability. Thus, we can say that allowing nodes to sleep during their active period, besides sleeping at the inactive period improves WBAN reliability, but at very high traffic rates, one should consider buffer overflow.

As for the end-to-end delay, Fig. 6 shows that Adaptive Sleep IEEE 802.15.4 results in a longer delay as opposed to the original IEEE 802.15.4 MAC due to the longer sleep period it offers. This is obvious at high and very high traffic rates. However, Adaptive Sleep's effect on the delay is almost identical to the legacy IEEE 802.15.4 MAC at low and moderate data packets rates, this is because the time of the occurrences of deep fade in the node's link is unknown. Indeed, not always the node need to sleep for the whole of its active duration, the node might sleep at the end of its active session if the fading occurs at the end of the nodes active duration, and in this case the effect of the delay at low and moderate traffic rate could be neglected. Either way, latency should always meet delay requirements as it is described in [1] [19], which is $250 \mathrm{~ms}$ at a maximum.

However, the longer sleep period offered by the Adaptive Sleep IEEE 802.15.4 helps in improving energy efficiency over the legacy IEEE 802.15.4 MAC, as the nodes will consume less energy and that's what Fig. 7 reveals. 


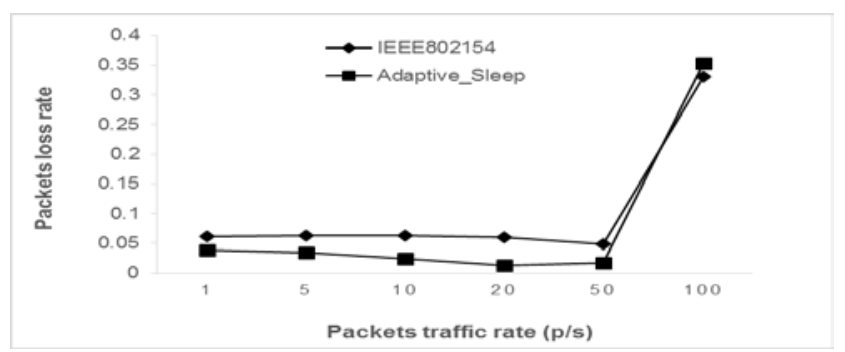

Fig. 5. Packets loss for an increasing packets rate

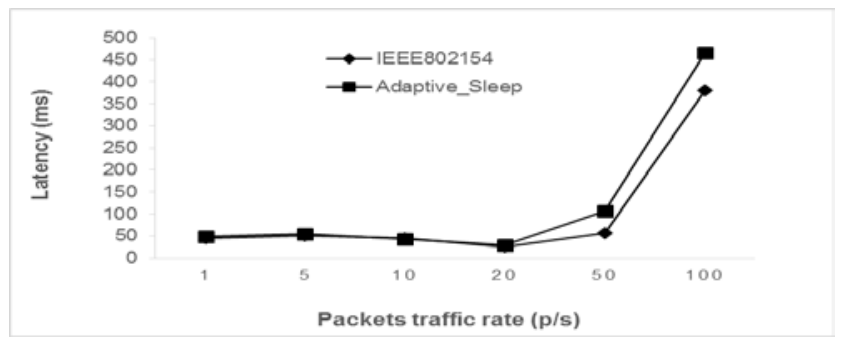

Fig. 6. End to end delay for an increasing packets rate

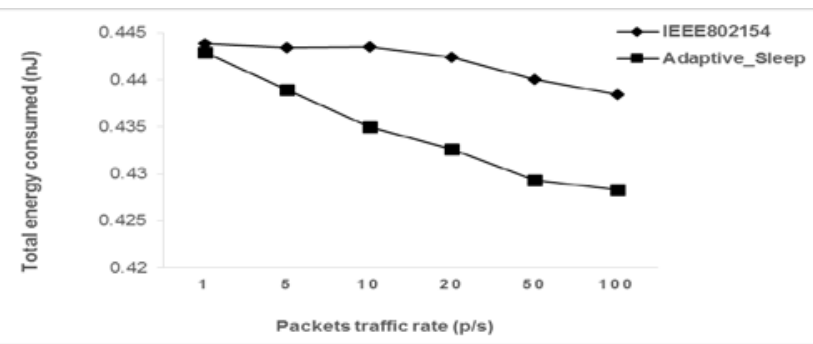

Fig. 7. Total energy consumed for an increasing packets rate

Adaptive Sleep causes frequent sleep periods during the active period of the superframe which increases packets latency and causes buffer overflow at very high data rates. Adaptive Sleep algorithm can be improved to fit various traffic rates by solving these issues, and this is why we proposed the second technique, which is presented in the following subsection.

\section{B. Dynamic GTS IEEE 802.15.4 MAC}

The drawback of buffer overflow can be solved by maximising the size of the buffer. However, this might be unfeasible solution for very tiny and resource constrained nodes. Therefore, other optimised solutions are required without the need to increase the size and the cost. Optimised solutions can be achieved by allocating the resources of nodes dynamically according to nodes needs. For example, extra slots can be allocated to those nodes that have more packets in their buffer than other ones. There is no need to perform any kind of computation to check how many packets in each node's buffer. Simply, nodes which have slept during their active period in the previous superframe would logically have more packets in their buffer than the others (assuming nodes transmit identical amount of traffic rates). However, as the number of time slots offered by IEEE 802.15.4 is limited to 16 time slots, the only way to give nodes extra slots is to borrow slots from the other nodes. In other words, time slots allocation will no longer be static. Time slot allocation is going to be dynamic according to nodes' needs.

Our proposed technique is based on the assumption that WBAN consists of 5 clients. Consequently, each node is allocated 3 time slots when the network is established. Then, during the network activities, those nodes that have slept adaptively during the current $\mathrm{BI}$ due to fading can be given extra slots in the next BI so they can have enough time to transmit what have been stored in their buffer in their current GTS(s). Those extra slots can be allocated from other nodes with links that have not suffered from fading in the current BI. This is to relax the pressure on their buffer and buffer overflow and to meet at the same time the delay requirement. The benefit of this solution should be noticed at the high traffic load networks. Moreover, the necessity of this solution becomes obvious in emergencies. The number of slots the node need to be allocated depends on the number of links encountered fading problem during the previous superframe. At its basic level, in this step, we excluded the context of the traffic, i.e. the level of traffic critically is the same for all nodes traffic and there is no traffic prioritization, and therefore we are dealing with a normal traffic of WBAN. Alternatively, the 16 time slots have been divided between the 5 nodes according to the following pattern:

- If one link faced fading, the node of that link will be reassigned 7 GTS(s), while the remaining nodes will be allocated 2 GTS(s).

- If two links faced fading: one node (of that link) will be assigned 5 GTS(s) and one 4 GTS(s) and nodes 2 GTS(s). The distribution of GTS(s) allocation depends on which node has slept first.

- If three links faced fading: two will be assigned 4 GTS(s) and one 3 GTS(s) while the rest of nodes 2 GTS(s). The distribution of GTS(s) allocation depends on which node have slept first.

- If four links faced fading: One will be assigned 4 GTS(s) and three 3 GTS(s) while one node 2 GTS. The distribution of GTS(s) allocation depends on which node has slept first.

- If five links faced fading: no change: all node will be assigned 3 GTS(s).

Nodes are re-allocated GTS(s) adaptively just when needed according to the occurrences of fading in the previous BI, which means that the pattern will be applied just on the next superframe. In the subsequent superframes, nodes will be reallocated a number of GTS(s) equivalent to what they asked for in their GTS(s) requests when the WBAN was established. This dynamic slot allocation pattern is merged with the Adaptive Sleep IEEE 802.15.4 technique presented in subsection IV-A and the overall algorithm now is called the Dynamic GTS. The pseudocode of the node's part of the algorithm is presented in in Algorithm 2. The pseudocode of the PANc's part is already depicted in Algorithm 1 that is presented in subsection IV-A.

One can notice that as the number of the faded links increases, the Dynamic GTS IEEE 802.15.4 might not cause much change in the original slot allocation pattern. It is worth stating however, that according to our simulated experiments, 
it is noticed that during all BIs, 3 nodes at maximum have faced fading. Thus, the last two cases have never occurred. We examined the performance of Dynamic GTS technique in the same topology presented in subsection IV-A and we followed the same configuration parameters.

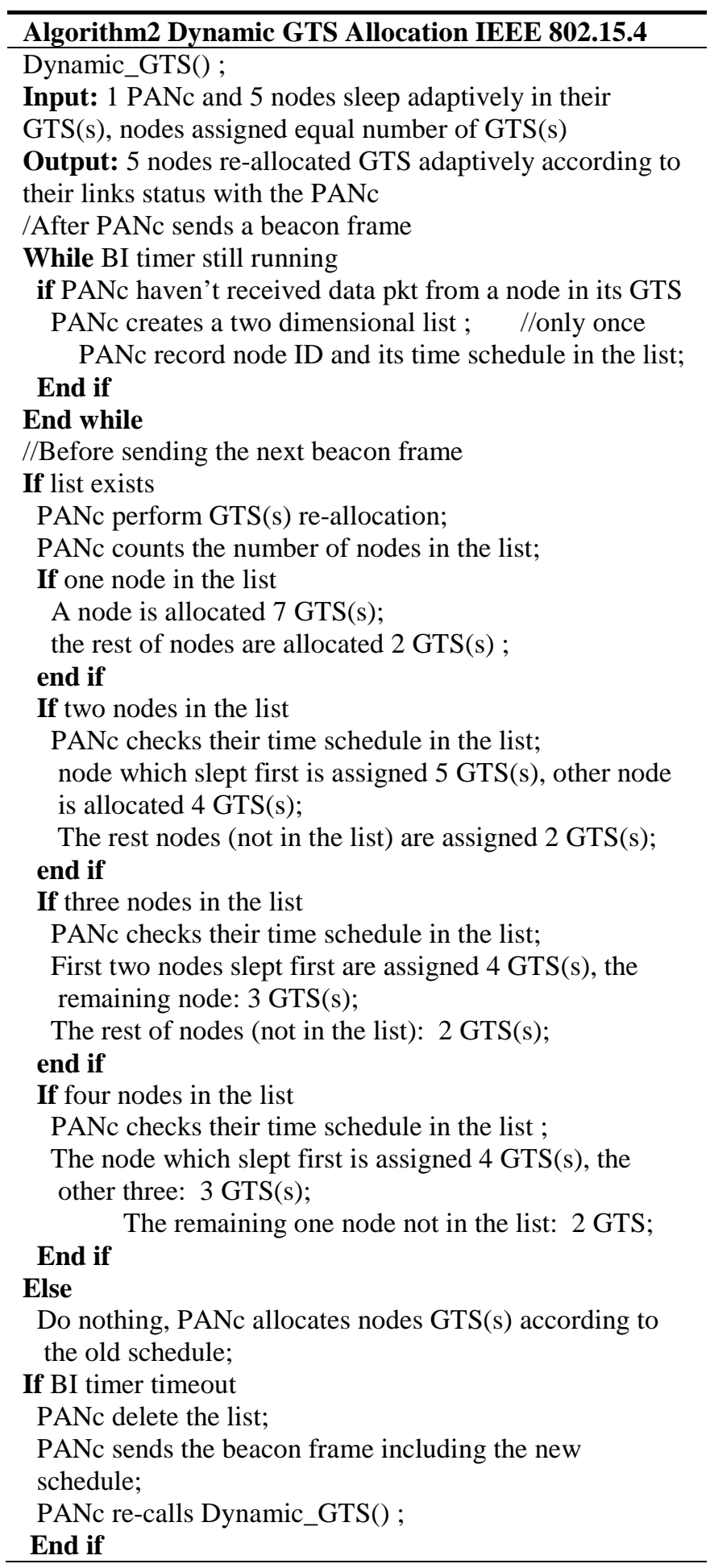

1) Performance Evaluation: The performance of the Dynamic GTS IEEE 802.15.4 is evaluated and compared with both the Adaptive Sleep and the legacy IEEE 802.15.4 MAC. Similar to subsections IV-A-2 and IV-A-3, the legacy IEEE 802.15.4 is first allowed to operate once within $25 \%$ duty cycle and once within $50 \%$ duty cycle. Because Adaptive GTS combines Adaptive Sleep technique, in this section, we refer to the Adaptive Sleep as Sleep only. First, we are going to discuss the results of comparing 50\% duty cycle Dynamic_GTS with both a 50\% duty cycle Adaptive_Sleep IEEE 802.15.4 and a 25\% duty cycle legacy IEEE802.5.4 MAC.

1) Scenario 1: 25\% duty cycle Legacy IEEE 802.15.4 versus. 50\% duty cycle Dynamic_GTS IEEE 802.15.4 MAC: We can see from Fig. 8 that, apart from the very high traffic loads, the Dynamic_GTS performance is almost identical to the Adaptive_Sleep IEEE 802.15.4 performance for all traffic loads. Packets loss that occurs at the very high traffic rates is due to the limited allocation slots assigned to nodes which's links did not face fading during the previous superframe (see slot allocation pattern). At those traffic rates, packets are not lost due to fading, they however, are lost due to buffer overflow. This calls for having a deeper insight at the proposed slot allocation policy. GTS(s) allocation strategy should be fair. Giving extra slots to nodes that suffered from fading during the previous superframe should not be at the expense of the performance of the nodes with good links status. Dynamic slot allocation should be based on some certain criteria that achieves both fair and reliable channel access at various traffic rates. One example to do so, is to perform a smart slot allocation based on the context of the traffic. Those nodes that send critical or emergent data should be assigned more slots than the other nodes that have normal or non-critical data, while considering the fading in the channel. Dynamic_GTS outperforms the legacy IEEE 802.15.4 due to the same reasons presented in subsection IVA-2. Actually, the legacy IEEE 802.15.4 allows nodes to sleep more than the other two algorithms and it does not consider the channel status, thus packets are lost due to both the vulnerability of the channel and due to buffer overflow, which's effect is obvious at very high data rates.

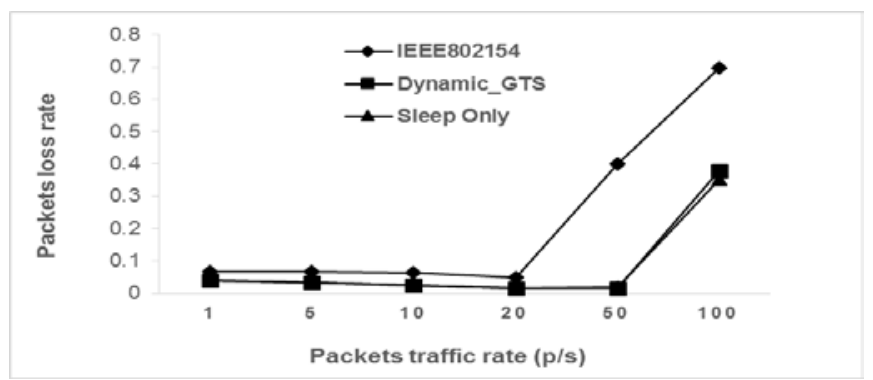

Fig. 8. Packets loss for an increasing packets rate

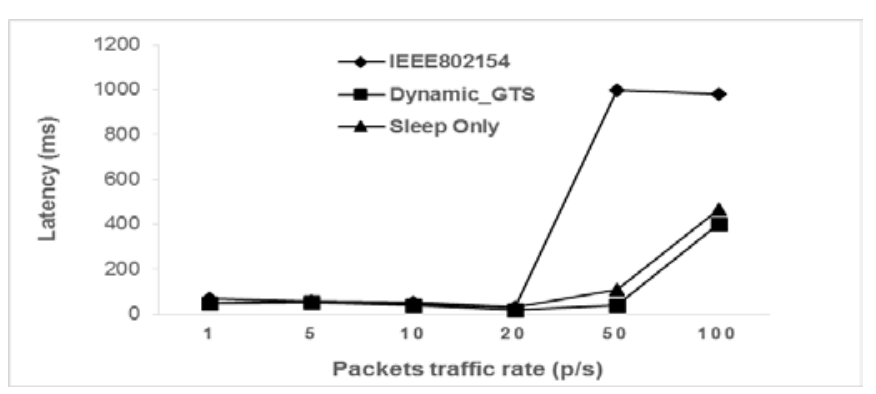

Fig. 9. End to end delay for an increasing packets rate 


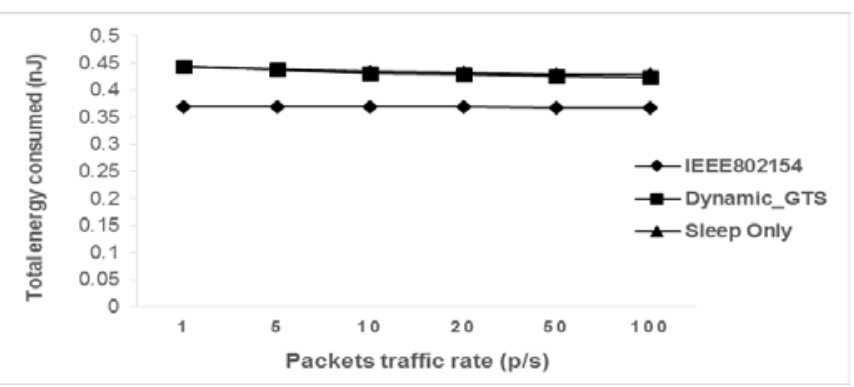

Fig. 10. Total energy consumed for an increasing packets rate

We can see from Fig. 9 that Dynamic_GTS performs better than both the Adaptive_Sleep and legacy IEEE 802.15.4 MAC in terms of the end-to-end delay at all the traffic rates. This is because nodes that saved their packets in the previous superfame, will have enough time to send their packets in the next superframe due to the extra slots that are acquired adaptively. This means that, in order to meet the WBAN delay requirements, it is worth performing dynamic slot allocation. The legacy IEEE 802.15.4 performs worse than the two algorithms due to the longer sleep period it offers ( $75 \%$ of the superframe structure). Long sleep period will not give nodes enough time to perform their activities at the current superframe, and nodes will differ sending their packets to the next superframe, which will increase delay especially at high and very high traffic rates.

When it comes to energy consumption, Fig. 10 reveals expected results, the legacy IEEE 802.15.4 performs better than the two proposed techniques in terms of energy consumption due to its longer sleep period, which will save more energy. However, the Dynamic_GTS performs slightly better than Adaptive_Sleep IEEE 802.15.4 in this regard. This is because besides allowing nodes to sleep in their active period, Dynamic_GTS offers nodes extra time slots just when they need, in order to relax the pressure on their buffers. Nodes with good links status however, are given limited number of slots, because they are not in need of extra slots as opposed to the other with bad links. Nodes with good links status are going to sleep instead of being idle at their time slots which would save energy.

2) Scenario 2: 50\% duty cycle Legacy IEEE 802.15.4 versus 50\% duty cycle Dynamic_GTS IEEE 802.15.4 MAC: Fig. 1113 show the result of comparing the Dynamic GTS with $50 \%$ duty cycle IEEE 802.15.4 (all algorithms operate within 50\% cycle and sleep for the rest $50 \%$ of the suprframe structure). Both the Adaptive Sleep and Dynamic GTS IEEE 802.15.4 allow the nodes to sleep further in their active period according to their link status as explained earlier.

Fig. 11 reveals that Dynamic GTS has comparable performance to Adaptive Sleep, while both outperform the legacy IEEE 802.15.4 MAC at all traffic rates apart from the very high traffic rates. This is because both Dynamic GTS and Adaptive Sleep IEEE 802.15.4 allow nodes to sleep more than the legacy IEEE802.15.4. This causes buffer overflow at very

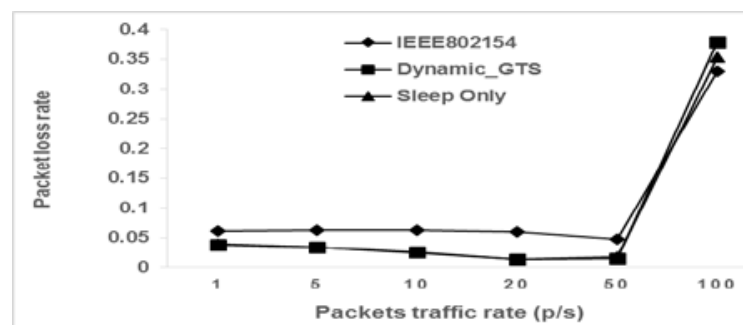

Fig. 11. Packets loss for an increasing packets rate

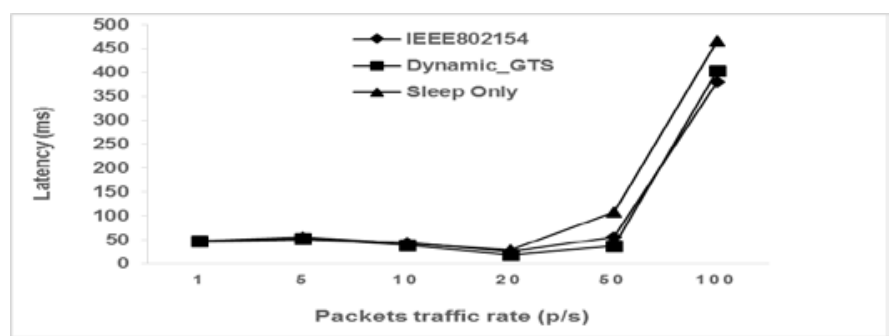

Fig. 12. End-to-end delay for an increasing packets rate

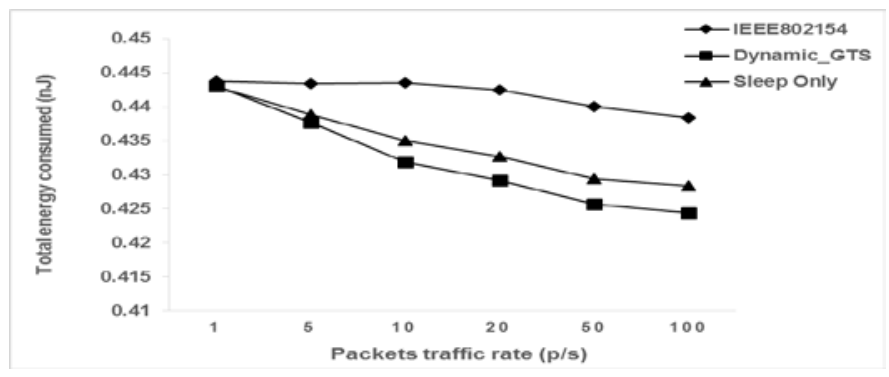

Fig. 13. Total energy consumed for an increasing packets rate

high traffic rates due to the limited size of the buffer. Dynamic GTS performs a bit worse than the Adaptive Sleep at very high traffic rates. This is because IEEE 802.15.4 offers only 16 slots, 15 of which are used as GTS(s), this leads to an unoptimal slot allocation, because Dynamic GTS improves the performance of those nodes with bad links in the previous superframs structure at the expense of the nodes with good links.

Regarding the end-to-end delay, Fig. 12 shows that Dynamic GTS reduces the end-to-end delay caused by Adaptive Sleep technique at all traffic rates. This is because it offers the nodes that slept during their previous active period enough time to send the packets that have been stored in their buffer. Dynamic GTS even outperforms the legacy IEEE 802.15.4 at moderate and high traffic rates though it allows the nodes to sleep more. However, it performs worse than the legacy IEEE 802.15.4 at very high data rates because more packets will be stored in the buffer for later transmission, which will increase latency at those buffered packets.

It is obvious from Fig. 13 that the Dynamic GTS algorithm consumes much less energy than both the Adaptive Sleep and the legacy IEEE 802.15.4 MAC especially at moderate, high and very high data rates. This is because not only Dynamic GTS algorithm allows nodes to sleep during their active period, but also it allocates nodes' slots according to their needs when there are other nodes with bad links at any BI. As a result, this will avoid idle listening for those nodes that do not need the three GTS slots. Extra slots will be given to those 
that need them urgently to relax the pressure in their buffer. This reduces the number of slots offered to nodes with good links and thus reduces their idle time. In brief, this technique will reduce energy dissipation and consequently improve the overall WBAN energy consumption.

\section{CONCLUSIONS AND FUTURE WORK}

WBAN reliability and energy efficiency can be improved by adopting a dynamic TDMA based channel access mechanisms that consider channel status. The two proposed techniques in this study are generic, as they do not require amendments to the legacy IEEE 802.15.4 specifications. As a future work, allocating time slots will be also based on the actual medical status of the nodes, which indicates whether the node in normal or emergency situation, this includes considering different types of applications, emergency and normal, downlink and uplink, proactive and reactive as well as different buffering techniques.

\section{REFERENCES}

[1] S. Movassaghi, M. Abolhasan, J. Lipman, D. Smith, and A. Jamalipour, "Wireless Body Area Networks: A Survey," Communications Surveys \&amp; Tutorials, IEEE, vol. 16, pp. 1658-1686, 2014.

[2] G. Wu, J. Ren, F. Xia, and Z. C. Xu, "An Adaptive Fault- Tolerant Communication Scheme for Body Sensor Networks," Sensors, vol. 10, pp. 9590-9608, 2010.

[3] S. Ullah, H. Higgins, B. Braem, B. Latre, C. Blondia, I. Moerman, et al., "A comprehensive survey of wireless body area networks," Journal of medical systems, vol. 36, pp. 1065-1094, 2012.

[4] H. R. Hongliang Ren and M. Q. H. Meng, "Understanding the Mobility Model of Wireless Body Sensor Networks," ed, 2006, pp. 306-310.

[5] S. Ullah, B. Shen, S. M. R. Islam, P. Khan, S. Saleem, and K. S. Kwak, "A Study of MAC Protocols for WBANs," Sensors (Basel, Switzerland), vol. 10, pp. 128-145, 12/28

[6] Z. Yan and B. Liu, "A context aware MAC protocol for medical Wireless Body Area Network," in Wireless Communications and Mobile Computing Conference (IWCMC), 2011 7th International, 2011, pp. 2133-2138.

[7] B. Liu, Z. Yan, and C. Chang Wen, "CA- MAC: A Hybrid contextaware MAC protocol for wireless body area networks," ed, 2011, pp. 213-216.

[8] S. C. Ergen, "ZigBee/IEEE 802.15. 4 Summary," UC Berkeley, September, vol. 10, p. 17, 2004.

[9] G. Z. Gang Zhou, J. L. Jian Lu, C.-Y. W. Chieh-Yih Wan, M. D. Yarvis, and J. A. Stankovic, "BodyQoS: Adaptive and Radio- Agnostic QoS for Body Sensor Networks," ed, 2008, pp. 565-573.

[10] Y. Tselishchev, A. Boulis, and L. Libman, "Variable Scheduling to Mitigate Channel Losses in Energy-Efficient Body Area Networks," Sensors, vol. 12, pp. 14692-14710, 2012.

[11] Y. Tselishchev, L. Libman, and A. Boulis, "Energy-efficient retransmission strategies under variable TDMA scheduling in body area networks," in Local Computer Networks (LCN), 2011 IEEE 36th Conference on, 2011, pp. 374-381.

[12] S. Rezvani and S. Ghorashi, "Context aware and channel- based resource allocation for wireless body area networks," IET Wireless Sensor Systems, vol. 3, pp. 16-16, 2013.

[13] Y. Yin, F. Hu, L. Cen, Y. Du, and L. Wang, "Balancing Long Lifetime and Satisfying Fairness in WBAN Using a Constrained Markov Decision Process," International Journal of Antennas and Propagation, vol. 2015, 2015.

[14] R. A. Tannious and A. Scaglione, "Physical layer design of distributed proportional fair scheduling in wireless body area networks," in Personal, Indoor and Mobile Radio Communications Workshops (PIMRC Workshops), 2010 IEEE 21st International Symposium on, 2010, pp. 78-83.

[15] B. Otal, C. Verikoukis, and L. Alonso, "Fuzzy-logic scheduling for highly reliable and energy-efficient medical body sensor networks," in
Communications Workshops, 2009. ICC Workshops 2009. IEEE International Conference on, 2009, pp. 1-5.S.

[16] Movassaghi, M. Abolhasan, J. Lipman, D. Smith, and A. Jamalipour, "Wireless Body Area Networks: A Survey," Communications Surveys \& Tutorials, IEEE, vol. 16, pp. 1658-1686, 2014.

[17] A. Boulis, Y. Tselishchev, L. Libman, D. Smith, and L. Hanlen, "Impact of wireless channel temporal variation on MAC design for body area networks," ACM Transactions on Embedded Computing Systems (TECS), vol. 11, p. 51, 2012.

[18] A. Boulis, D. Smith, D. Miniutti, L. Libman, and Y. Tselishchev, "Challenges in body area networks for healthcare: The MAC," Communications Magazine, IEEE, vol. 50, pp. 100-106, 2012.

[19] A. Boulis and Y. Tselishchev, "Contention vs. polling: A study in body area networks MAC design," in Proceedings of the fifth international conference on body area networks, 2010, pp. 98-104.

[20] M. Maman, B. Denis, and R. D'Errico, "Research trends in wireless body area networks: From on-body to body-to-body cooperation," in Medical Information and Communication Technology (ISMICT), 2014 8th International Symposium on, 2014, pp. 1-5.

[21] H. Su and X. Zhang, "Battery-dynamics driven TDMA MAC protocols for wireless body-area monitoring networks in healthcare applications," Selected Areas in Communications, IEEE Journal on, vol. 27, pp. 424434, 2009.

[22] A. El-Hoiydi, J.-D. Decotignie, C. Enz, and E. Le Roux, "WiseMAC, an ultra low power MAC protocol for the wiseNET wireless sensor network," in Proceedings of the 1st international conference on Embedded networked sensor systems, 2003, pp. 302-303.

[23] O. Omeni, A. Wong, A. J. Burdett, and C. Toumazou, "Energy efficient medium access protocol for wireless medical body area sensor networks," Biomedical Circuits and Systems, IEEE Transactions on, vol. 2, pp. 251-259, 2008

[24] S. J. Marinković, E. M. Popovici, C. Spagnol, S. Faul, and W. P. Marnane, "Energy-efficient low duty cycle MAC protocol for wireless body area networks," Information Technology in Biomedicine, IEEE Transactions on, vol. 13, pp. 915-925, 2009.

[25] G. Fang and E. Dutkiewicz, "BodyMAC: Energy efficient TDMA-based MAC protocol for wireless body area networks," in Communications and Information Technology, 2009. ISCIT 2009. 9th International Symposium on, 2009, pp. 1455-1459.

[26] N. F. Timmons and W. G. Scanlon, "An adaptive energy efficient MAC protocol for the medical body area network," in Wireless Communication, Vehicular Technology, Information Theory and Aerospace \& Electronic Systems Technology, 2009. Wireless VITAE 2009. 1st International Conference on, 2009, pp. 587-593.

[27] I. T. G. (TG4). (2003, Sunday, 31 January 2016). IEEE 802.15 WPAN Task Group 4 (TG4). Available: http://www.ieee802.org/15/pub/TG4.html

[28] A. Boulis, "Castalia: A simulator for wireless sensor networks and body area networks," NICTA: National ICT Australia, 2011. 